\title{
Editorial: Structural and computational glycobiology - immunity and infection
}

\author{
Mark Agostino ${ }^{1,2 *}$ and Elizabeth Yuriev ${ }^{3 *}$ \\ ${ }^{1}$ CHIRI Biosciences and Curtin Institute for Computation, School of Biomedical Sciences, Curtin University, Perth, WA, Australia, \\ ${ }^{2}$ Centre for Biomedical Research, Burnet Institute, Melbourne, VIC, Australia, ${ }^{3}$ Medicinal Chemistry, Monash Institute of \\ Pharmaceutical Sciences, Monash University, Melbourne, VIC, Australia
}

Keywords: glycobiology, structural biology, infection, cancer immunotherapy, molecular modeling, molecular recognition, lectins, signaling

Historically deemed as the realm of the brave or the foolhardy, glycobiology has grown considerably as a discipline over the last 50 years. Carbohydrates, which were once considered to be mere "decorations" on proteins and lipid membranes, are increasingly demonstrated to afford specific roles in signaling and communication (1).

Although the rate of structures deposited into the Protein Data Bank continues to grow at an exponential rate, the characterization of new structures of carbohydrate-protein complexes is growing more modestly, still being very challenging and prone to errors (2). Computational methods are increasingly being pursued to provide structural insight into carbohydrate-protein interactions. The complex structure and high flexibility of carbohydrates, as well as difficulties associated with accurately computing binding energies for these interactions, present considerable challenges for the

OPEN ACCESS

Edited and reviewed by:

Kendall Arthur Smith Weill Medical College of Cornell University, USA

*Correspondence: Mark Agostino mark.agostino@curtin.edu.au; Elizabeth Yuriev

elizabeth.yuriev@monash.edu

Specialty section:

This article was submitted to Immunotherapies and Vaccines, a section of the journal

Frontiers in Immunology

Received: 19 June 2015 Accepted: 01 July 2015 Published: 14 July 2015

Citation:

Agostino M and Yuriev E (2015) Editorial: Structural and computational glycobiology immunity and infection. Front. Immunol. 6:359. doi: 10.3389/fimmu.2015.00359 use of these methods in both understanding the carbohydrate-protein recognition and the structureaided design of carbohydrate-based therapeutics. However, numerous computational approaches have been developed in recent years that address some of these issues (3-9). The Opinion piece in this Research Topic further highlights some computational resources that have been developed specifically for glycobiology (10).

Several carbohydrate classes, most notably gangliosides, Lewis antigens, and ThomsenFriedenreich antigen, are of considerable interest for the development of cancer immunotherapeutics. Krengel and Bousquet (11) present a comprehensive review on the importance of gangliosides not only to cancer therapeutics but also their relevance for signaling and in mediating infection by pathogens, as well as how their structure and presentation on glycolipids and glycoproteins influences their function and potential to be exploited in therapeutics. Ahmed et al. (12) describe the use of molecular modeling to optimize framework regions of an anti-ganglioside antibody, resulting in the identification of a new construct with enhanced stability, antigen binding, and cytotoxic properties. Kieber-Emmons et al. (13) discuss the challenges and frontiers associated with the development of peptides as immunogenic mimics of carbohydrates, particularly focusing on mimics of tumor-associated carbohydrate antigens.

Despite considerable advances in the understanding of many aspects of glycobiology, several fundamental processes remain only partially understood. An excellent example of this is the structural basis of antibody recognition of the blood group antigens (A, B, H). Makeneni et al. (14) combine docking with a recently developed carbohydrate-specific scoring function and molecular dynamics simulation to demonstrate the structural basis of A vs. B specificity of an anti-A antibody. Lee et al. (15) performed LC-MS/MS-based glycomics and proteomics, combined with structural analyses, of a wide range of glycosylated proteins in order to understand the differences in the glycosylation of secreted cell surface and intracellular proteins. The study correlates the presence of specific $N$-glycan terminations with their subcellular location, providing insight into pathophysiological conditions 
caused by glycosylation disorders. Brockhausen (16) provides a comprehensive review detailing known glycosyltransferases with overlapping activities between bacteria and mammals. In many cases, similar catalytic mechanisms between bacterial and mammalian glycosyltransferases can be identified, despite limited sequence similarity.

Lectins, particularly C-type lectins, are of considerable importance for immunity, mediating cell-cell recognition, and representing potential targets for the development of therapeutics. Notable C-type lectins include DC-SIGN and the selectins, known for their roles in the progression of HIV and cancer, respectively. Richardson and Williams (17) review the discovery and characterization of the macrophage C-type lectin (MCL) and

\section{References}

1. Varki A. Essentials of Glycobiology. 2nd ed. Cold Spring Harbor, NY: Cold Spring Harbor Laboratory Press (2009).

2. Agirre J, Davies G, Wilson K, Cowtan K. Carbohydrate anomalies in the PDB. Nat Chem Biol (2015) 11:303. doi:10.1038/nchembio.1798

3. Agostino M, Mancera RL, Ramsland PA, Yuriev E. AutoMap: a tool for analyzing protein-ligand recognition using multiple ligand binding modes. J Mol Graph Model (2013) 40:80-90. doi:10.1016/j.jmgm.2013. 01.001

4. Tessier MB, Grant OC, Heimburg-Molinaro J, Smith D, Jadey S, Gulick AM, et al. Computational screening of the human TF-glycome provides a structural definition for the specificity of anti-tumor antibody JAA-F11. PLoS One (2013) 8:e54874. doi:10.1371/journal.pone.0054874

5. Kerzmann A, Fuhrmann J, Kohlbacher O, Neumann D. BALLDock/SLICK: a new method for protein-carbohydrate docking. J Chem Inf Model (2008) 48(8):1616-25. doi:10.1021/ci800103u

6. Eid S, Saleh N, Zalewski A, Vedani A. Exploring the free-energy landscape of carbohydrate-protein complexes: development and validation of scoring functions considering the binding-site topology. J Comput Aided Mol Des (2014) 28:1191-204. doi:10.1007/s10822-014-9794-3

7. Agostino M, Yuriev E, Ramsland PA. Antibody recognition of cancer-related gangliosides and their mimics investigated using in silico site mapping. PLoS One (2012) 7:e35457. doi:10.1371/journal.pone.0035457

8. Pérez S, Sarkar A, Rivet A, Breton C, Imberty A. Glyco3D: a portal for structural glycosciences. Methods Mol Biol (2015) 1273:241-58. doi:10.1007/ 978-1-4939-2343-4_18

9. Kirschner KN, Yongye AB, Tschampel SM, González-Outeiriño J, Daniels CR, Foley BL, et al. GLYCAM06: a generalizable biomolecular force field. Carbohydrates. J Comput Chem (2008) 29:622-55. doi:10.1002/jcc. 20820

10. Yuriev E, Ramsland PA. Carbohydrates in cyberspace. Front Immunol (2015) 6:300. doi:10.3389/fimmu.2015.00300 the macrophage-inducible C-type lectin (Mincle), their roles in initiating the immune response to infection, and the identification of activating ligands for these receptors. Aretz et al. (18) predict the druggability of a panel of C-type lectins, as well as perform fragment-based screening by nuclear magnetic resonance spectroscopy against DC-SIGN, langerin, and MCL. Their work highlights limitations in the application of computational methods to predict the druggability of this class of proteins.

The work presented in this Research Topic illustrates a small selection of the wide ranging research in this area and the considerable challenges associated with both understanding glycan function and targeting glycan interactions for the development of therapeutic agents.

11. Krengel U, Bousquet PA. Molecular recognition of gangliosides and their potential for cancer immunotherapies. Front Immunol (2014) 5:325. doi:10.3389/ fimmu.2014.00325

12. Ahmed M, Hu J, Cheung N-K. Structure based refinement of a humanized monoclonal antibody that targets tumor antigen disialoganglioside GD2. Front Immunol (2014) 5:372. doi:10.3389/fimmu.2014.00372

13. Kieber-Emmons T, Pashov A, Saha S, Monzavi-Karbassi B, Murali R. Carbohydrate mimetic peptides for pan anti-tumor responses. Front Immunol (2014) 5:308. doi:10.3389/fimmu.2014.00308

14. Makeneni S, Ji Y, Watson DC, Young NM, Woods RJ. Predicting the origins of anti-blood group antibody specificity: a case study of the ABO A- and B-antigens. Front Immunol (2014) 5:397. doi:10.3389/fimmu.2014.00397

15. Lee LY, Lin C-H, Fanayan S, Packer NH, Thaysen-Andersen M. Differential site accessibility mechanistically explains subcellular-specific N-glycosylation determinants. Front Immunol (2014) 5:404. doi:10.3389/fimmu.2014.00404

16. Brockhausen I. Crossroads between bacterial and mammalian glycosyltransferases. Front Immunol (2014) 5:492. doi:10.3389/fimmu.2014.00492

17. Richardson MB, Williams SJ. MCL and mincle: C-type lectin receptors that sense damaged self and pathogen associated molecular patterns. Front Immunol (2014) 5:288. doi:10.3389/fimmu.2014.00288

18. Aretz J, Wamhoff E-C, Hanske J, Heymann D, Rademacher C. Computational and experimental prediction of human C-type lectin receptor druggability. Front Immunol (2014) 5:323. doi:10.3389/fimmu.2014.00323

Conflict of Interest Statement: The authors declare that the research was conducted in the absence of any commercial or financial relationships that could be construed as a potential conflict of interest.

Copyright (c) 2015 Agostino and Yuriev. This is an open-access article distributed under the terms of the Creative Commons Attribution License (CC BY). The use, distribution or reproduction in other forums is permitted, provided the original author(s) or licensor are credited and that the original publication in this journal is cited, in accordance with accepted academic practice. No use, distribution or reproduction is permitted which does not comply with these terms. 